\title{
Impact of a nationwide COVID-19 lockdown on acute coronary syndrome referrals
}

\author{
Erik W. Holy, Philipp Jakob, Robert Manka, Barbara E. Stähli, \\ Patrick T. Siegrist, Frank Ruschitzka, Christian Templin \\ University Heart Center Zurich, Switzerland \\ University Hospital Zurich, Switzerland
}

Editorial p. 478

Since December 2019, the emergence of coronavirus disease 2019 (COVID-19) in Wuhan, China, has evolved towards a global pandemic stressing healthcare providers and local authorities all over the world [1]. While national coronavirus lockdowns have led to a deferral of elective procedures, the European Society of Cardiology (ESC), has issued guidance for the diagnosis and management of cardiovascular diseases during the COVID-19 pandemic, in particular acute coronary syndromes (ACS). However, reports recently published suggest a decline in primary percutaneous coronary intervention (PCI) volumes during the COVID-19 pandemic $[2,3]$. In addition to these observations, we report the immediate impact of a nationwide lockdown during the COVID-19 outbreak on ACS referrals in a tertiary care center as well as data on death tolls in Switzerland during the observation period.

Acute coronary syndrome patients and out of hospital cardiac arrests (OHCA) referred to the catheterization laboratories at the University Heart Center Zurich during the period 02/17-04/12/2020 were included. The number of ACS referrals reported 4 weeks before and after implementation of a nationwide lockdown on March $16^{\text {th }} 2020$ was compared to the same period of time in 2019.

Four weeks after March $16^{\text {th }} 2020$ ACS referrals decreased by $42 \%$ (non-ST-segment elevation myocardial infarction: $-49 \%$, ST-segment elevation myocardial infarction: $-56 \%$, unstable angina: $+37 \%$ ) while OHCA referrals declined by $57 \%$ (Fig. 1A). An initial decrease in ACS referrals was observed following the first report of a COVID-19 case in Switzerland on February $25^{\text {th }}$ ( -2 weeks) and was precipitated by the implementation of a nationwide lockdown on March $16^{\text {th }}$ (Fig. 1C). Numbers of ACS and OHCA referrals remained stable for the same observation period in 2019 (Fig. $1 \mathrm{~B}, \mathrm{D})$. The decline in ACS referrals observed from March $16^{\text {th }} 2020$ on was paralleled by an increase in weekly reported deaths in the population of persons aged 65 and over in Switzerland (Fig. 1E) while death numbers remained unchanged in 2019. Exposure to air pollutants is associated with an increased risk of near-term myocardial infarctions [4]. To assess the effect of restrictive actions following implementation of a lockdown on urban air quality, the time evolution of atmospheric pollutants recorded at a traffic air quality monitoring station were analyzed. Temperature monitoring revealed a significant increase in local temperature following the lockdown in $2020\left(+2.2^{\circ}\right.$, $\mathrm{p}=0.02$, Fig. 2A). As compared to 2019 an overall reduction in nitric oxides, pollutants mainly related to traffic emissions (Fig. 2B) could be observed during the COVID-19 pandemic. However, no significant difference in nitric oxides levels was observed before or after March $16^{\text {th }}$. On the other hand, an increase in atmospheric particulates (PM10, Fig. 2C) was be registered for both years following March $16^{\text {th }}$ which is most probably related to the prevailing secondary origin of fine aerosols as well as seasonal Sahara dust contributions.

The present study supports previous observations and demonstrates a dramatic drop in ACS referrals within a few weeks during the COVID-19 pandemic in a tertiary care center in Switzerland which was precipitated by the implementation of a nationwide lockdown. Despite the potential

Address for correspondence: Erik W. Holy, MD, PhD, University Heart Center Zurich, Rämistrasse 100, 8091 Zurich, Switzerland, tel: +41 4425521 21, e-mail: erik.w.holy@gmail.com 


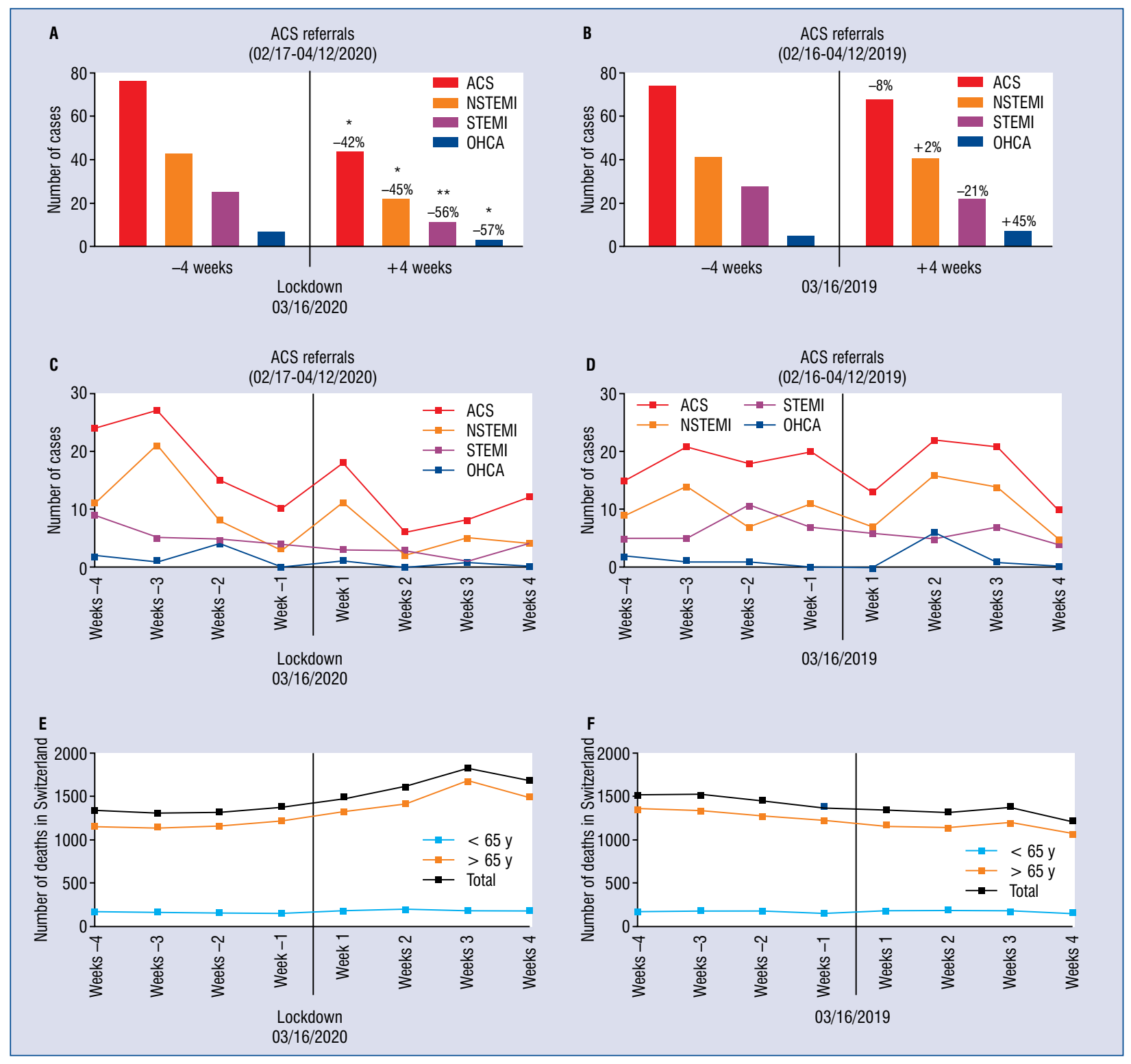

Figure 1. Acute coronary syndrome (ACS) referrals 4 weeks before and after March $16^{\text {th }} 2020$ (A) and 2019 (B). Weekly evolution of ACS referrals for the same period of time 2020 (C) and 2019 (D); ${ }^{*} p=0.02$ and ** $p<0.01$ for +4 weeks versus -4 weeks. Report of death cases in Switzerland according to the Federal Office of Public Health (published 04/21/2020) for the study period 2020 (E) and 2019 (F); NSTEMI — non-ST-segment elevation myocardial infarction; STEMI - ST-segment elevation myocardial infarction; OHCA — out of hospital cardiac arrest; y — years.

beneficial effects observed on traffic related air pollution, environmental changes do not seem to explain the extent of this decline in ACS referrals. The latter is paralleled by a nationwide increase in deaths observed during the pandemic in the population aged 65 and over as compared to the same period in 2019. Considering the growing evidence suggesting a strong contribution of cardiovascular mechanisms in COVID-19 associated complications, the same concerns are shared by our colleagues regarding the fear of on an increase in overall mortality due to a high rate of untreated ACS during the pandemic [5, 6]. While administrations and healthcare systems need to continue to consider all measures necessary to contain outbreaks, campaigns in order to avoid taking a toll on other medical urgencies beyond immediate infections, in particular ACS are mandatory.

Conflict of interest: None declared 


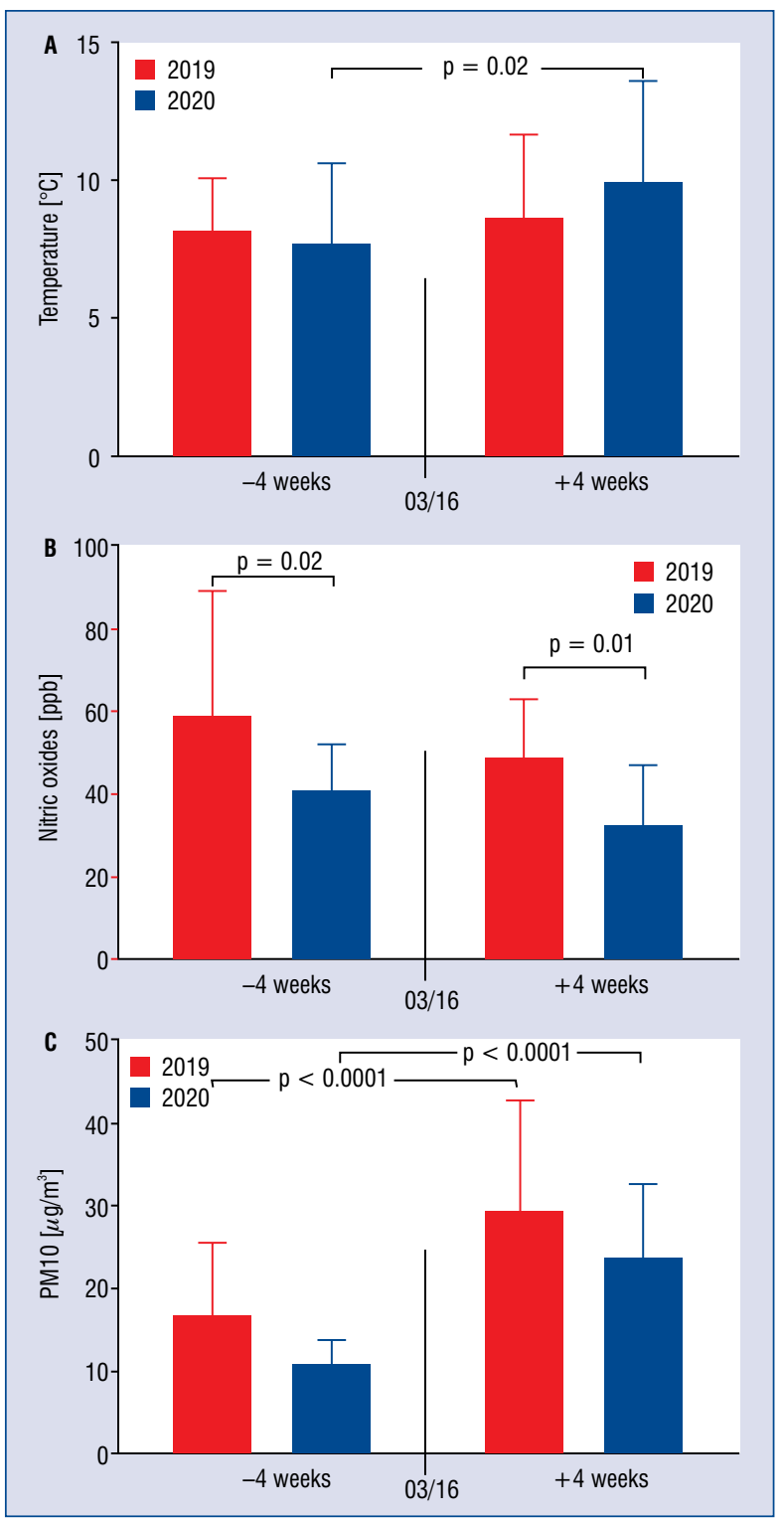

Figure 2. Analysis of temperature (A), nictric oxides (B) and particulates (PM10; C) variations at the traffic air quality monitoring station Rosengartenstrasse, Zurich, Switzerland. Data provided by the Office of the Environment (www.ostluft.ch). Statistical analysis was performed using two-way ANOVA (GraphPad Prism 6.0). Bars display mean values and standard deviations.

\section{References}

1. Studdert DM, Hall MA. Disease control, civil liberties, and mass testing - calibrating restrictions during the COVID-19 pandemic. N Engl J Med. 2020 [Epub ahead of print], doi: 10.1056/ NEJMp2007637, indexed in Pubmed: 32272003.

2. Metzler B, Siostrzonek P, Binder RK, et al. Decline of acute coronary syndrome admissions in Austria since the outbreak of COVID-19: the pandemic response causes cardiac collateral damage. Eur Heart J. 2020; 41(19): 1852-1853, doi: 10.1093/ eurheartj/ehaa314, indexed in Pubmed: 32297932.

3. De Rosa s, Spaccarotella C, Basso C, et al. Reduction of hospitalizations for myocardial infarction in Italy in the COVID-19 era. Eur Heart J. 2020; 41: 2083-2088.

4. Mustafic H, Jabre P, Caussin C, et al. Main air pollutants and myocardial infarction: a systematic review and meta-analysis. JAMA. 2012; 307(7): 713-721, doi: 10.1001/jama.2012.126, indexed in Pubmed: 22337682.

5. Xiong TY, Redwood S, Prendergast B, et al. Coronaviruses and the cardiovascular system: acute and long-term implications. Eur Heart J. 2020; 41(19): 1798-1800, doi: 10.1093/eurheartj/ ehaa231, indexed in Pubmed: 32186331.

6. Varga Z, Flammer AJ, Steiger P, et al. Endothelial cell infection and endotheliitis in COVID-19. Lancet. 2020; 395(10234): 1417-1418, doi: 10.1016/S0140-6736(20)30937-5, indexed in Pubmed: 32325026. 